\title{
Study of Drypoint Transfer Value and Technical apply on Linen
}

\author{
Liao Jiangbo \\ College of Textiles, Donghua University \\ Jiang Xi Institute of Fashion Technolgoy ${ }^{2}$ \\ Shanghai, China \\ E-mail: 284704383@qq.com
}

\author{
Yang Xiaoming \\ College of Humanities, Donghua University \\ Shanghai, China \\ E-mail:ynide@sina.com* \\ Corresponding Author: Yang Xiaoming
}

Keywords: Drypoint; Linen; Transferprinting; Ethching; Couture

\begin{abstract}
Etching belongs to an ancient creative art of Europe, and it originated gold silversmith printing process in European. Copper plate has a certain strength, through the copperplate etching and release agent transfer printing plate making, printing on linen, you can get the printing design creative nature. Etchings of transfer printing on linen, provided a feasibility scheme of flax printing DIY accessories and haute couture.
\end{abstract}

Transfer printing is a printing process, the creation engravings are using pressure printing method of release agent. Linen on transfer printing process is common laser transfer printing, thermal transfer and silk screen printing. They have their own advantages and disadvantages of various kinds of transfer printing technology, and adapt to different production requirements. Photoengraving transfer printing, laser transfer printing and heat transfer, is to spin out the patterns and background as a whole, after the transfer printing on textiles. They have obvious drawbacks, namely, easy to cause transfer part of airtight, water absorption while moisture is slow, perspiring all over. Silk screen printing is now the most common means of transfer printing, T-shirts produced using tinted way of transfer printing, when the color is too much for the color pattern plate making process many corresponding complex, transfer printing costs will rise. At present, the transfer printing on linen, the domestic research has Gao Shuzhen [1], Sun Hongyan [2], Wang Changrong [3], and so on, they discuss the linen dyeing and printing techniques. Experimenting with a new way of transfer printing: creative etchings of transfer printing on linen. Etchings on linen printing technology research, can improve the cultural quality of flax printing, lacking the ability to try to solve the pattern design and printing, the quality is not high. Combined with stylist character printing at the same time, the realization of smallscale production, for the enterprise and Independent designers with a printing solutions.

\section{Several Kinds of Transfer Printing Technology Advantages}

Domestic textile character pattern transfer printing production basically points mode of four categories: silk screen printing, thermal transfer, embroidery and hand draw. Screen printing is a kind of the most widely used way of pattern making [4], and printing technology is mature, the market $90 \%$ of personality $\mathrm{T}$-shirts are screen printing, screen printing to leak edition technology, mainly divided into technical process, design, film, plate making, printing, dry several steps. Screen printing using matching color printing, if a design on the T-shirts are divided into red, yellow, blue, and black four colors, it needs to print the four edition, each color one version. Silk screen printing mainly can be divided into: water slurry printing, rubber paste printing, and ink printing. Silk screen printing by added to some special material, can make a difference in printing effect to meet customer's special requirements. Thermal transfer printing is a printing technology, popular in these years is to print or printed onto a transfer printing paper, through the high temperature and high pressure on the shirt fabric, the pattern of relative silk screen printing, transfer printing with fast and convenient, the advantages of vivid, but also relatively low color fastness, mass customization cost higher shortcomings. Suitable for small amount of personalization. Thermal transfer printing, suitable for 
small area large printing on textiles, close to the skin will feel uncomfortable [5]. The embroidery can be divided into manual and computer embroidery, high cost, complex process, the embroidery LOGO as a general brand LOGO. Hand-painted, market application is not much, some consumers with handpainted DIY show personality.

Creative prints as a pure arts, printing on textiles have corresponding threshold, business professionals to be engaged in engravings in the studio to do transfer printing t-shirts, but is only individual behavior, and transfer printing quality is not high, there is no systematic study.

\section{Character Printing Design Market Forecast and Research Value}

Personality Printing Design Market Forecast. Clothing personality a trend and tendency of the development of printing design, because modern people like to make public self, reflect their own personality, which makes that linen shirt is sought after by more and more people, especially young people. General derivative of t-shirts for DIY T-shirt, DIY is to Do it. For the designs in the T-shirts are the wearer himself selected and then processing printed on clothes, so call DIY. Also gradually rise in DIY concept formation, a related peripheral industry, more and more people began to think about how to make DIY into their lives. Creative prints on the linen shirt transfer embodies is a kind of DIY, it can satisfy the consumer to hand the devotion of individual character, at the same time can also be a small amount of replication. Other fashionable clothing design and art design, is a designer and behavior of the individual. Although drypoint transfer printing technology is still not popularized in the market, but occupy a leading style in designer brands main position of age, an important part of a niche market, it can enrich, rich apparel market, at the same time fashion DIY concept allows designers to creative design into product design.

At present in China, based on the perspective of culture, the original did a lot of attempts to retrofit design of the green clothing design, old clothes, quilts, semi-finished products design, costume design hand-painted with embroidery design, etc. In China, most of the colleges and universities of apparel arts , crafts and graphically creative use separate teaching model, clothing handicraft bead embroidery, embroidery lack of graphic originality. However, there are creative design and crafts conjointly good case. Suzhou university college of art and design creative ,it design and product design the teaching mode of combining clothing professional students through the creative graphic appears in the form of handicrafts in garment accessories, a flea market to get students in this school. Zhejiang university of technology institute of fashion technology established t-shirts design studio to relate design and handmade, through the school business incubators sell DIY dress shirt. The concept of DIY recreates the students' creativity, it is in line with teaching ideas from creative design to product design, and also will more and more attention of the school.

Introducing drypoint $\mathrm{T}$-shirts production embodies the culture of linen shirt, originality, if printmaking simplification can be solved, the quality of the images in the process of transfer printing, environmental protection, color fastness and other issues, so creative prints transfer in t-shirts can yet be regarded as an effective way. The key technology in DIY printmaking technology research. A simple print production, can let experience master engraving production skills. Experience in the creation of the concept of DIY printmaking sense of accomplishment, experience manual labor to bring emotional enjoyment and satisfaction. Writing on the paper transfer pattern have certain interest, culture and fashion.

\section{Research Value}

a) We research of Nanchang city in Jiangxi province, Jiangxi province is the largest jute spinning, is also a big T-shirts and export processing province. Qingshan lake area of more than 3000 small clothing in the home, there are more than 1200 enterprises engaged in the production of T-shirt. Insufficient capacity of apparel pattern design, and insufficient capacity of knitting fabric printing and dyeing, High levels of water to wash, embroidery, printing matching ability is insufficient. Creative prints on T-shirts printing technology research can improve the cultural quality of the T-shirt, lacking the ability to try to solve the pattern design and printing, the quality is not high. 
b) The economic benefits of creative print t-shirts. 1) DIY manual pieces, let everybody become designer provides a realistic way. In some special functions, it can be printed on t-shirts customers like pictures, make customers feel new, as a kind of consumption with gifts, to experience the fun of cultural consumption. (2) To cater to consumers in the pursuit of original works. At present, on the linen T-shirt design creation, with photos and copy images as linen T-shirt design, originality is not enough. (3) Of the project economic estimates. A pure linen T-shirt production cost and material cost price in 25 yuan, linen shirt t-shirts after processing market price is in 100 yuan, if the print t-shirts printed by hand the price is in 200 yuan, then creating print t-shirts premium is in 100 yuan, if the monthly sales volume in 1000, then can create a monthly profit of 100000 . On the investment of equipment, equipment investment in 1000 yuan of letterpress engravings, intaglio etching equipment investment about 30000 or so, leaking plate silk screen engraving equipment at about 20000 yuan.

c) Social benefits. Advocate green clothing design, and promote DIY costume culture in the society. Costume design professionals to promote to the society, the designer can effectively and graphically creative achievements into designs. In addition, this topic research can also let students produce part of the economic benefits, upload some mature DIY works to the school of business incubators Taobao shop to sell, or trade directly in the school.

\section{Research and Development Content, Methods and Technical Route}

The Raw Material of Choice. Raw materials selection, 2001 water double unbleached linen (Figure 1) and Marley brand oil paintings, linen main properties index of yarn weave $10.5 \mathrm{~N} * \mathrm{~N}$, 10.5 44/36 warp/weft density. Print as a picture, has double attribute of the replication and uniqueness, the primitive form of printed on the paper become a print. Then, transfer printing on textiles, the difference between experimental textile this kind of material with paper, including the pressure in the gravure printing, printing ink selection and configuration, as well as color fastness, image transfer printing quality and related issues. Internationally, flax textiles is regarded as the symbol of human civilization, it is widely used in clothing, decoration and other fields. Because of flax fiber structure is tight, high crystallinity, orientation degree and the causes of small Angle dyeing printing is more difficult. [6] However, oil paints are used as a "stain", there is a larger advantage. Oil paints by pigment powder added flaxseed oil and rubber mixing grinding and become, linen with linseed oil and glue from the same kind of plants, it is not easy to fade. Linen, made from linen twist thread, has the vivid texture and is easy to color.

Considering the high price of copper, the use of zinc plate (Figure 2) instead of copper plate, zinc and copper have similar chemical and physical properties. Understandably, the medium of the copper plate, zinc plate printed on international printmaking collectively known as etchings. $0.2-0.4 \mathrm{~mm}$ thick industrial zinc plate, and coated with blue paint on the back are chosen, it can prevent people from by nitric acid corrosion in the process of plate making.

Figure 1. Linen 


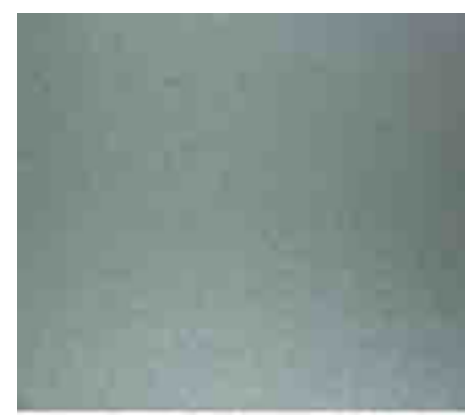

Figure 2. Zinc Plate
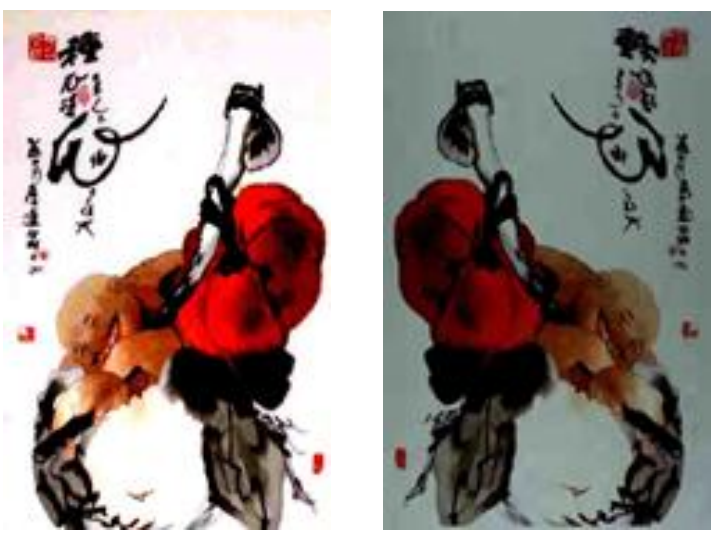

Figure 3. Transfer Patterns Figure 4. Patterns Transfer Printing on the Copper

The Choice of Transferring Printing Design. Transfer printing design is transferring printing on linen printing design, it should be the pursuit of creative prints of culture and fashion. Prints are works of art, there is a certain difference with the pattern on the t-shirts. Image creation art performance extremely strong artistic quality, design is rich, but the design requires marked on $\mathrm{T}$ shirts, fashion and popularity. Under the influence of printing process, the creation prints should take into account the simplicity in plate making, too delicate transfer out effect. Pattern area is not too big, too many images can affect the whole clothes permeability, and perspiration is not easy to discharge, so the picture can be white. For example, Figure 3:

Figure 3 linen figure 2 zinc plate Figure 3 transfer printing design

Copper Plate Heat Transfer Process. Will Figure 3 with banana oil, medical alcohol, No. 90 gasoline as release agent transfer printing on the copper plate to the original, and then a certain percentage of the modulation good nitric acid and water, as an etchant. During the dry line, corrosion, dust, soft wax, such as comprehensive processing technology, is different from the original pictures and images, with the creation of the creative nature of prints. Specific operation process is as follows:

a) With sand paper burnish a flat or charcoal first zinc, not let zinc have a scratch;

b) Read the pictures posted on the surface of dry zinc, tape, is for the sake of the jie, layout does not move;

c) Put on a mask and gloves, flat with the version of the picture, the banana oil or rosin water on the cloth. Cloth is used to dip in taking rosin water or banana oil to wipe on the back of the picture. Can use toilet paper to do the cloth shape (because toilet paper has the character of water absorption), outside the toilet paper again to find a few compare wear-resisting layer of cloth outside, and then bound to strong. Note that not pour too much at a time, more can't poured banana oil or rosin water directly on the image (lacquer thinner and rosin water are chemicals, solvent is used to dilute the harmonic agent. Pour in the picture on the one hand will put the picture on the carbon powder diluted, but can't transfer printing on the board, on the one hand was transfer to board of carbon powder due to banana oil or rosin water penetration, again transfer up and transfer printing down); 
d) With rapidly scoured, in the picture on the back of the cloth for rosin water and banana oil volatilization is faster. The best look, while wiping, ensure that the toner transfer printing to the version of Figure 3. Note that the backlight place can be observed;

e) Copper plate on micro electric furnace heating, saw smoke should be stopped immediately, when indicated toner is attached on the copper plate;

f) In the mixed nitric acid corrosion liquid corrosion. If required heavy color, can be more than 2 minutes, or within 2 minutes. Simple explain here, if on this basis, the daub on the paint, wipe again, can get Figure 3 reversal film as shown in Figure 4.

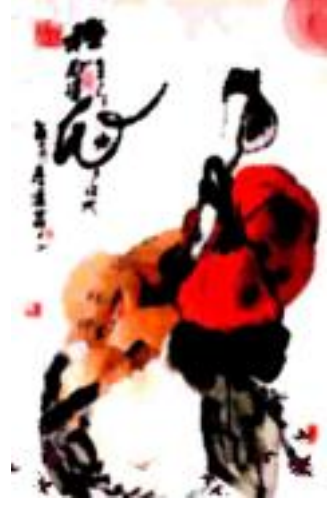

Figure 5. Designed Good Sketch

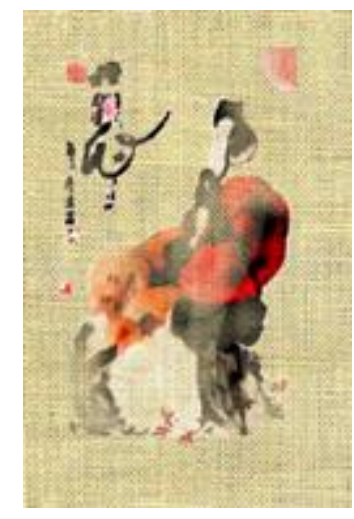

Figure 6. Transfer printing Effect Ion

g) On the corrosion good print, can be dealt with again. First of all, do a simple with Figure 3, add red leaves and the full moon, and the effect of fly ash, simulation design draft Figure 5. Particular way, in the layout of coated with a thin layer of anti-corrosion membrane, and then use a needle or knives needed to score on screen image, add the nitric acid corrosion in the solution, after a score and corroded place form recessed grooves line or block surface, after wash anticorrosive film, painted image is clearly displayed on the page; [7]

Figure 4 transfers printing patterns on copper plate Figure 5 design sketch Figure 6 transfer printing effect Ink

h). Will be transfer printing and the corrosion of the oil paints squeeze into the concave part of line and the surface of the block. In advance will need oil paints, in ordinary white paper, 24 hours a day, let the inside of the oil paints linseed oil and vegetable oil, omit part of such pigment will appear dry. When the clean version, should not be erased, there will be less and peculiar smell;

i) Wiping away excess oil paints. Will not out of line and the surface of the block of oil paints to wipe clean, inside to gently wipe, should not be excessive, safeguard the image clear; Brush (10) pressure. Put the linen on copper plate on stamping, inhale oils after embossing machine printing on linen. Prior to stamping of linen fabrics, water first, then slowly dry, surface is wet, but it can't squeeze out water;

j) Dry. Remove pressure printed linen, linen "prints", namely is drypoint design creation. As shown in Figure 6.

4) Pay Attention to the Problems

Oil paints particles of fly ash techniques of loose powder particles and how much how much, the effect of toner many candle out of deep color, texture is more exquisite, images appear thicker. Toner less corruption candle out the color of the shallow, also rawer sugar, picture looks thin. The experiment suggests the application of the toner can be artificially controlled, can need according to picture to adjust the dosage of the toner. What toner and its thickness of image transfer printing effect is very big, we can use in the creation process of the change of the difference to control and to create images. Print pictures and decay by transfer to the version of the candle compared to printed images, effect is also different. Because in the process of heat transfer, due to the pressure and flux of water (or banana oil) with different concentration, the quality of the after transfer printing image difference. [8] 
The etchings on linen printing has a great chance. Drypoint make itself has a lot of chance, some factors cannot be controlled by human. Nitrate is easy to volatilize, affects the effect of corrosion on the strength of the nitric acid solution. Mechanism of linen, will affect the adhesion effect of oil paint on it. The weight of the clean version, which will influence the copper plate imaging. Even, air humidity, vegetable oil and flax oil content in the oil paints, will affect the final result. The contingency, also let etchings of transfer printing on linen with extremely strong artistic quality.

\section{Conclusion}

From relief to gravure are through design pattern to plate making, silk screen version, coloring, transfer printing, drying such a series of process. Creative prints of transfer printing on linen is a process that combines an integrated manual and painting, making the process of stress ability, not only have painted, still can use scissors, stick, carved, to roll printing, printing, coating, touch, pressure, etc., which use a lot of tools, has a strong technology and artistic quality. Image transfer printing plate-making technology on the copper plate. Photos without agent and computer image design, transfer to the copper plate, then the image is obtained by corrosion prototype, the final version will corrosion coating ink transfer printing on t-shirts. The development and research, the main study of rapid reproduction, the computer software design of digital images can be applied to the traditional print plate making, increase the artistic quality of the image. On the basis of experiments, the research of new material, new process transfer printing technology, expand the etching technique, explore new image printing technology, seeking the best computer made to the amount of image pixels, ink transfer agent in the process of plate making the image quality.

\section{REFERENCES}

[1] Gao Shuzhen. Ultrasonic application (DOIs): in flax fabric dyeing printing and dyeing, 2000 (12).

[2] Sun Hongyan etc. To improve flax fiber pad dyeing dyeing chemical and adhesive performance research (DOIs): 2005 (05).

[3] Wang Changrong. High-grade practice color bleaching process of linen fabric in study (DOIs): heilongjiang textile, 2015 (01).

[4] Lin Chengkun. Silkscreen printing technology research. China packaging engineering, 2001 (6) : pp. 13 - 17.

[5] Yan Suzhai. Heat transfer techniques (DOIs): screen printing, 2001 (6)

[6] Liu Jie,Gao Jie. Flax fabricpigment printing process(DOIs):

journal of qiqihar university, 2012 (12) : 20 -22.

[7] Wei-xin Wang. Drypoint techniques research new art, 1983 (04) : 39-44.

[8] Zhang Peng-guo. Etchings of transfer printing techniques applied research [D] xi 'an academy of fine arts graduate dissertation, 2012 (3) : 8.

[9] Wang Yanfeng. The creation of etchings language. yanbian university graduate dissertation, 2013 (05) : 20-32. 